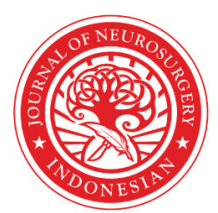

\section{Jugular foramen lesion diagnostic dilemma - schwannoma or paraganglioma: A case report}

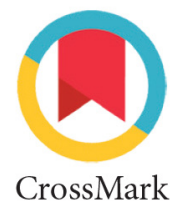

\author{
Sachin Sambhaji Chemate ${ }^{1 *}$, Joy Varghese ${ }^{2}$, Chandrasekar K², \\ Shankar Ganesh CV², Mangaleswaran Balamurugan ${ }^{2}$
}

\title{
ABSTRACT
}

Introduction: Various anomalies occure in the jugular foramen. The most common lesion within the jugular foramen is the hypervascular glomus jugulare tumour followed by neurogenic tumors, predominantly schwannoma. Jugular foramen schwannoma can also be vascularized and frequently mimics paraganglioma.

Case presentation: We present a case of a 36-year-old woman who presented with 8 to 10 months history of giddiness and vertigo, slowly progressive sensorineural hearing loss in left ear, gradually progressive left facial weakness (House Brackman grade III at the time of admission) and gait ataxia. A magnetic resonance imaging showed an avidly enhancing extra axial left cerebellopontine angle space occupying lesion extending through the jugular foramen into the petrous temporal bone on the left side with prominent central flow voids. Paraganglioma was suspected and Digital Subtraction Angiography was done which showed tumour blush with arteriovenous fistula supplied by stylomastoid artery, which is branch of occipital artery. Surprisingly, histopathology after surgical resection revealed schwannoma.

Conclusion: Prominent central vascularity and arteriovenous fistula with arterial supply from stylomastoid branch of occipital artery can be an unusual presentation for jugular foramen schwannomas. We describe architecture of vascualrity of jugular schwannoma and identify stylomastoid branch of occipital artery as its prime feeder.

Keywords: jugular foramen schwannoma, paraganglioma

Cite This Article: Chemate, S.S., Varghese, J., Chandrasekar, K. Ganesh, S.C.V., Balamuragan, M. 2019. Jugular foramen lesion diagnostic dilemma - schwannoma or paraganglioma: A case report. Indonesian Journal of Neurosurgery 2(3): 76-79. D01: 10.15562/ijn.v2i3.51

${ }^{1}$ Resident, Department of Neurosurgery, Apollo Hospitals, Chennai

${ }^{2}$ Senior Consultant, Department of Neurosurgery, Apollo Hospitals, Chennai

*Corresponding author: Sachin Sambhaji Chemate; Resident, Department of Neurosurgery, Apollo Hospitals, Chennai; sachin.19chemate@gmail.com

Received: 2019-01-22

Accepted: 2019-02-03

Published: 2019-12-01

\section{INTRODUCTION}

A diverse group of vascular anomalies originate in the jugular foramen..$^{1-4}$ The most common tumour within the jugular foramen is the hypervascular glomus jugulare tumour followed by neurogenic tumors, predominantly schwannoma. ${ }^{5,6}$ Less common lesions comprise meningioma, hemangiopericytoma, chondrosarcoma, and plasmacytoma. Metastases and malignant tumours arising in adjacent anatomic structures (nasopharynx, parotid, and temporal bone) in advanced stages may spread to the jugular foramen.

Schwannomas involving the jugular foramen are rare neoplasms and very few cases have been reported in the literature. ${ }^{78}$ Usually, it is misdiagnosed as acoustic neuroma and the diagnosis is made perioperatively. ${ }^{9,10}$ Schwannomas are relatively avascular but jugular foramen schwannoma is known to be hypervascular neoplasm. It has significant neurological morbidity and mortality. ${ }^{11-13}$

Paraganglioma is a vascular lesion with the ascending pharyngeal artery as a main feeder. The jugular foramen schwannoma is also hypervascular lesion like paraganglioma but very little information available in literature about the architecture of its vascularity and no single feeding artery has been identified for schwannoma in literature. The aim of this study is to identify the architecture of vascularity and blood supply of the jugular foramen schwannoma.

\section{CASE PRESENTATION}

We present a case of a 36-year-old lady from northern part of India, known case of type II diabetes. She presented with history of giddiness and vertigo since 10 months, gait imbalance since 8 months with tendency to fall on left side, slowly progressive sensorineural hearing loss in left ear, gradually progressive left facial weakness since 3 - 4 months, difficulty in swallowing liquids and change of voice since 1 month.

On examination she was conscious and oriented. There was no papilledema. She had signs of left cerebellar hemisphere dysfunction with left VII nerve palsy (HB grade III), left ear sensorineural hearing loss, dysphagia for liquids and impaired left gag reflex suggestive of left IX and X nerve 
impairment. Trapezius and sternocleidomastoid muscle bulk was normal but weak on left side.

Based on history and examinations, jugular foramen paraganglioma was suspected. Brain Magnetic Resonance Imaging (MRI) with contrast was done which showed an extraaxial mass measuring $6.5 \times 5.4 \times 3.6 \mathrm{~cm}$ in left cerebellopontine angle space extending through the jugular foramen into the petrous temporal bone on the left side which was hypointense on T1W and hyperintense on T2W and FLAIR with heterogeneous contrast enhancement with salt and paper appearance (Figure 1).

As paraganglioma was considered as first differential diagnosis and supported by brain MRI that was suggestive of a vascular lesion, the patient underwent Digital Subtraction Angiography

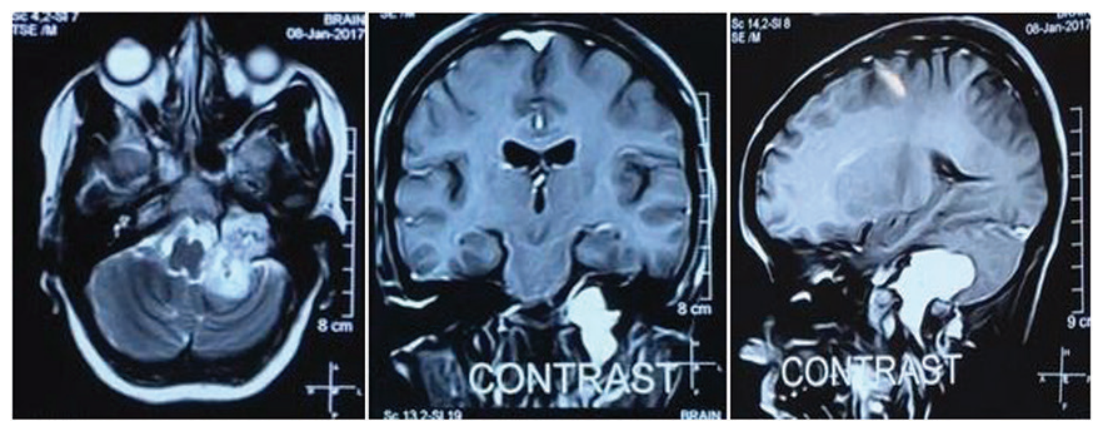

Figure 1. Brain MRI with GADO showing left cerebellopontine angle space occupying lesion extending through the jugular foramen into the petrous temporal bone on the left side, showing heterogeneous contrast enhancement with salt and paper appearance.
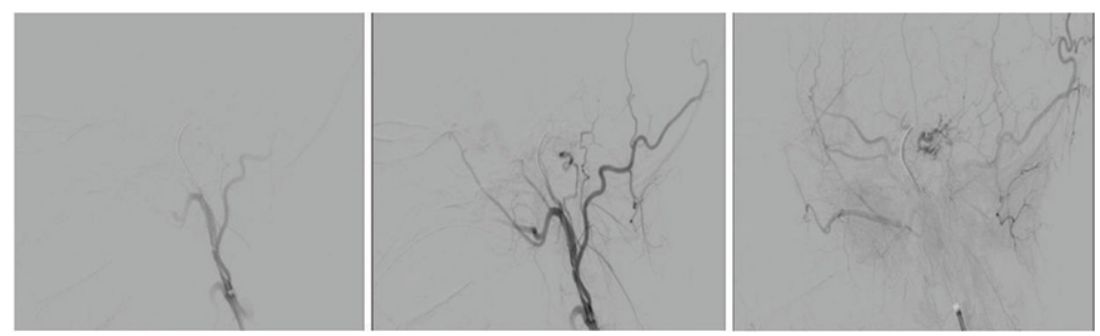

Figure 2. DSA showing tumour blush with arteriovenous fistula.
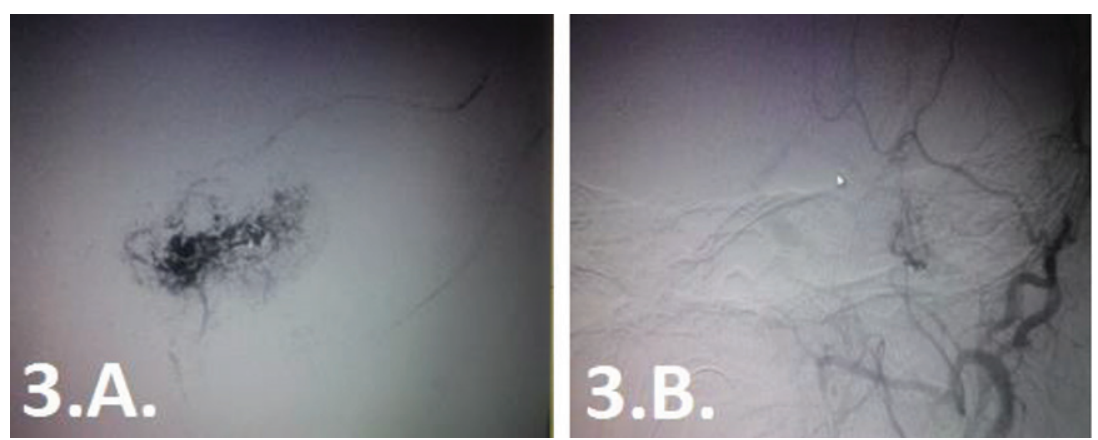

Figure 3. A) Lateral view showing early filling of draining vein though a fistula, B) Early feeling of draining vein even before appearance of tumor blush, suggestive of arteriovenous fistula.
(DSA) which revealed a tumour blush in the region of jugular foramen which was suggestive of a hypervascular lesion (Figure 2). There was a draining vein which was seen before appearance of tumor blush which was suggestive of an arteriovenous fistula (Figure 3A and 3B). Only one isolated feeder of arteriovenous fistula was identified. In unsubtracted view, the same feeding vessel was seen from stylomastoid foramen (Figure 4B), hence stylomastoid artery (branch of occipital artery) was identified as the primary feeder of lesion and the cause of arteriovenous shunting (Figure 4A) in this case (and not by the ascending pharyngeal artery which is the known feeder of glomus jugulare).

Arteriovenous shunting seen on DSA was more suggestive of paraganglioma. Hence, we decided performed combined approach with preoperative embolization followed by craniotomy and excision of lesion. Preoperative embolization of feeding vessel was done. Post embolization, there was no tumor blush and fistula was completely obliterated.

Patient underwent surgery post embolization. We used combined approach with translabyrynthine petrosectomy with left presigmoid and retrosigmoid craniotomy. Intraoperatively, tumour was highly vascular inspite of preoperative embolization. Complete excision of lesion was done. Postoperatively patient had left side sensorineural hearing loss, left facial palsy, and dysphagia. She was discharged on postoperative day $7^{\text {th }}$ with Ryle's tube feeding. She was gradually started on speech and swallowing therapy.

Histopathological examination showed fragments of spindle cell lesion composed of predominantly hypercellular areas with elongated bland nuclei and moderate eosinophilic cytoplasm (Antoni A) at places and hypocellular areas containing myxoid (Antoni B) were also seen. Verrocay bodies were evident. Few aggregates of fomy histiocytes and cystic changes were noted. Focal lymphoid infiltrates were evident. Focal degenerative changes were also seen. Variable vascular density were noted composed of both thick and thin walled at foci showing proliferating vessels. This histomorphological features were consistent with schwannoma (WHO grade I) (Figure 5).

At 6 month of follow up, brain MRI with contrast showed small residual lesion measuring $1.2 \times 1.1 \mathrm{x}$ $0.9 \mathrm{~cm}$ (Figure 6) which was managed with single fraction cyberknife therapy.

\section{DISCUSSION}

In present day, development of medical science has provided us better understanding of various disease in cerebello-pontine angle and jugular 

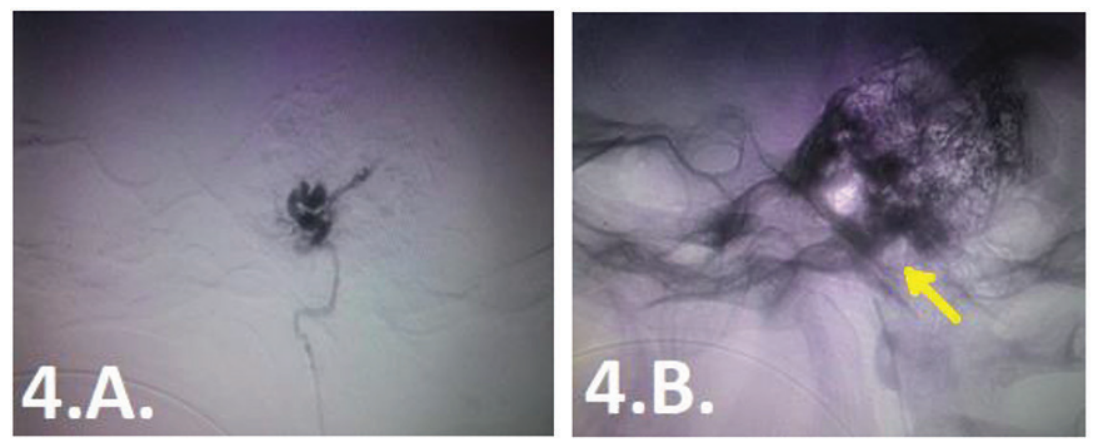

Figure 4. A) Stylomastoid artery feeding the tumour, B) Arrow shows the artery in stylomastoid foramen in unsubstracted view.
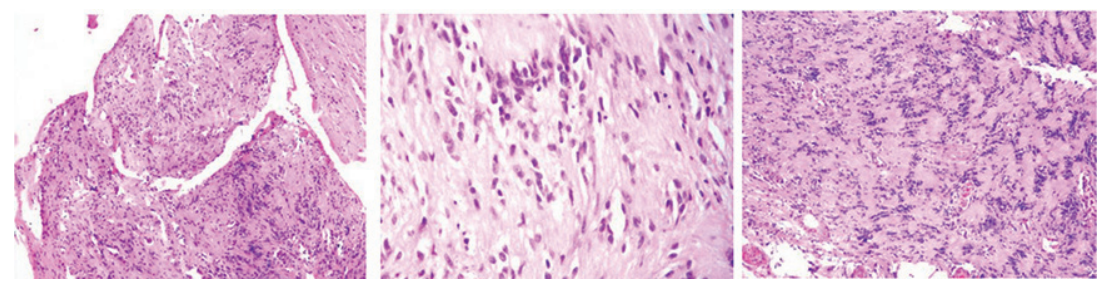

Figure 5. HPE showing schwannoma (WHO grade I)
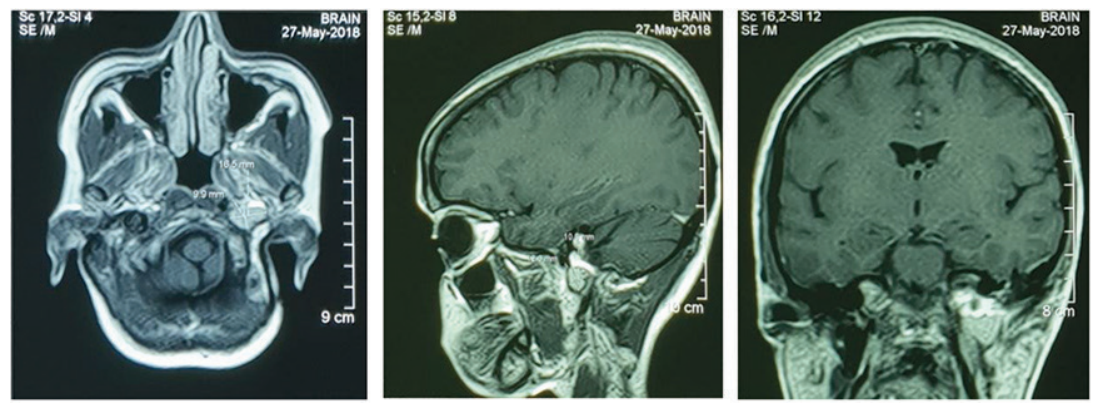

Figure 6. Post op scan at 6 month follow up showing small residual lesion

fossa. ${ }^{1-4}$ Whenever a vascular lesion was identified in jugular foramen, then paraganglioma was the suspicion. ${ }^{5,6}$ Schwannomas elsewhere are relatively avascular or hypovascular on angiography. However, jugular foramen schwannomas are known to be hypervascular and very few cases have been reported in the literature..$^{7-14}$ Lee $\mathrm{M}$, et al $^{13}$ stated that, although hypervascular paraganglioma is most common lesion in jugular foramen, jugular schwannoma can present or mimic as paraganglioma. Ascending pharyngeal artery has already been described and proven to be the prime feeder of hypervascular paraganglioma. ${ }^{5-6}$

However, some case report argue that ascending pharyngeal artery is also prime feeder of arteriovenous shunting seen in jugular foramen schwannoma. ${ }^{8,9,11-13}$ However, in our case, a stylomastoid branch of occipital artery is the actual feeder of arteriovenous shunting (Figure 4A, 4B). We propose that the pathophysiology of VII nerve palsy is not due to mechanical compression but it is because of ischemia due to shunting from stylomastoid branch of occipital artery.

Schwannomas are typically described as avascular or hypovascular on angiography. We describe an unusual case of a jugular foramen schwannoma with blood supply from stylomastoid branch of occipital artery. To our knowledge this is the first case reported in the literature to talk about blood supply of jugular schwannoma, though we need more cases to support the evidence.

\section{CONCLUSION}

Prominent central vascularity and arteriovenous fistula within the tumour can be an unusual presentation of jugular foramen schwannomas. We describe architecture of vascularity of jugular schwannoma and identify stylomastoid branch of occipital artery as its prime feeder. We also propose that the pathophysiology of VII nerve palsy is not due to mechanical compression but it is because of ischemia due to shunting from stylomastoid branch of occipital artery.

\section{AUTHOR'S CONTRIBUTION}

All authors contribute equally in the collection, analysis, and interpretation of data. All authors have read and agreed to the final manuscript.

\section{CONFLICT OF INTEREST}

There is no conflict of interest regarding this article.

\section{FUNDING}

There is no sponsorship regarding this article.

\section{REFERENCES:}

1. Yousem DM, Grossman RI. Neuroradiology: The Requisites. 3rd ed. Philadelphia, Mosby; 2010.

2. Caldemeyer KS, Mathews VP, Azzarelli B, Smith RR. The jugular foramen: a review of anatomy, masses, and imaging characteristics. Radiographics. 1997; 17(5): 1123 - 1139. DOI: 10.1148/ radiographics.17.5.9308106.

3. Vogl TJ, Bisdas S. Differential diagnosis of jugular foramen lesions. Skull Base. 2009; 19(1): 3 - 16. DOI: 10.1055/s-0028-1103121.

4. Ramina R, Maniglia JJ, Fernandes YB, Paschoal JR, Pfeilsticker LN, Neto MC, et al. Jugular foramen tumors: diagnosis and treatment. Neurosurg Focus. 2004; 17(2): E5. DOI: 10.3171/foc.2004.17.2.5.

5. Rao AB, Koeller KK, Adair CF. From the archives of the AFIP. Paragangliomas of the head and neck: radiologic-pathologic correlation. Armed Forces Institute of Pathology. Radiographics. 1999; 19(6): 1605 - 1632. DOI: 10.1148/ 
radiographics.19.6.g99no251605.

6. Olsen WL, Dillon WP, Kelly WM, Norman D, Brant-Zawadzki M, Newton TH. MR imaging of paragangliomas. AJR Am J Roentgenol. 1987; 148(1): 201 - 204. DOI: 10.2214/ajr.148.1.201.

7. Eldevik OP, Gabrielsen TO, Jacobsen EA. Imaging findings in schwannomas of the jugular foramen. AJNR Am J Neuroradiol. 2000; 21(6): 1139 -1144 .

8. King CD, Long JM, Hammon WM. Early draining veins in acoustic neurinomas. Radiology. 1974; 113(2): 369 - 371. DOI: 10.1148/113.2.369.

9. Moscow NP, Newton TH. Angiographic features of hypervascular neurinomas of the head and neck. Radiology. 1975; 114(3): 635 - 640. DOI: 10.1148/114.3.635.

10. Kato H, Kanematsu M, Mizuta K, Aoki M, Kuze $\mathrm{B}$, Ohno T, et al. "Flow-void" sign at MR imaging: a rare finding of extracranial head and neck schwannomas. J Magn Reson Imaging. 2010; 31(3): 703 - 705. DOI: 10.1002/jmri.22071.
11. Bonneville F, Cattin F, Czorny A, Bonneville JF. Hypervascular intracisternal acoustic neuroma. $J$ Neuroradiol. 2002; 29(2): 128 - 131.

12. Yamakami I, Kobayashi E, Iwadate Y, Saeki $\mathrm{N}$, Yamaura A. Hypervascular vestibular schwannomas. Surg Neurol. 2002; 57(2): 105 - 112. DOI: $10.1016 / \mathrm{s} 0090-3019(01) 00664-4$

13. Lee $\mathrm{M}$, Tong K. Jugular foramen schwannoma mimicking paraganglioma: case report and review of imaging findings. Radiol Case Rep. 2016; 11(1): 25 - 28. DOI: 10.1016/j.radcr.2015.12.002.

14. Samii M, Babu RP, Tatagiba M, Sepehrnia A. Surgical treatment of jugular foramen schwannomas. J Neurosurg. 1995; 82(6): 924 - 932. DOI: 10.3171/ jns.1995.82.6.0924.

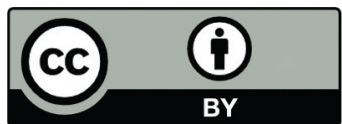

This work is licensed under a Creative Commons Attribution 